\title{
Stab-wounds and other penetrating injuries of the abdomen and thorax
}

\author{
J. B. GARLAND \\ M.B., F.R.C.S. \\ Surgical Registrar, \\ Manchester Royal Infirmary, Manchester 13
}

\section{Summary}

A series of 100 penetrating wounds of the abdomen and thorax is reviewed. Eighty-three were due to stab-wounds.

The recent increase in incidence is confirmed and the distribution throughout the day and months of the year is given.

The results and methods of treatment of thirtysix abdominal, ten thoraco-abdominal and thirtyseven thoracic wounds are given and described.

An operative approach to abdominal wounds is supported.

Conservative measures, including more frequent use of intercostal-tube drainage for pneumothorax and haemothorax, are satisfactory for the majority of chest wounds.

Cardiac wounds require urgent assessment and resuscitation with pericardiocentesis and thoracotomy.

\section{Introduction}

Stab-wounds are occurring with increasing frequency in urban hospital practice in Great Britain (Editorial, 1966). There are few British reports on this type of injury, in marked contrast to their frequent appearance in American literature. For this reason the present study was undertaken with a view to assessing the incidence, the presenting symptoms and signs and the results of treatment.

\section{Material}

One hundred cases of penetrating injury of the abdomen and thorax treated at the Manchester Royal Infirmary over the period 195666 were analysed. Eighty-three of these cases were due to stab-wounds.

\section{Incidence}

The trend in the yearly increase in stabwounds is illustrated in Fig. 1. Prior to 1960 the incidence was five or less annually, between 1960 and 1964 this had doubled, and in the years 1965 and 1966 this had doubled again.
No corresponding increase is seen in the incidence of other penetrating wounds of the abdomen and thorax.

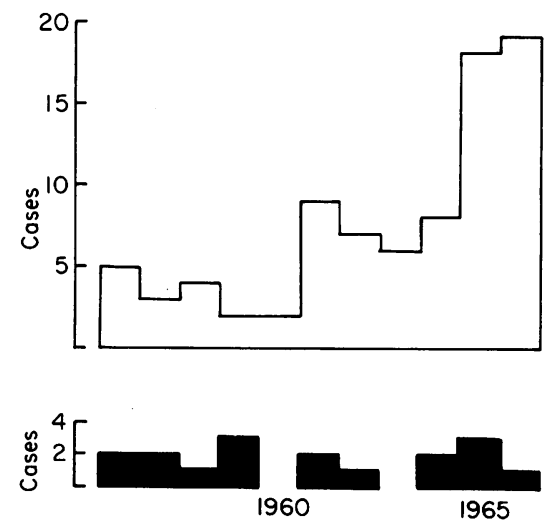

FIG. 1. Yearly incidence of wounding. Upper histogram: incidence of stab-wounds; lower histogram: incidence of other penetrating injuries.

An analysis of the distribution of these stabwounds throughout the months of the year (Fig. 2) showed a fairly even distribution. A
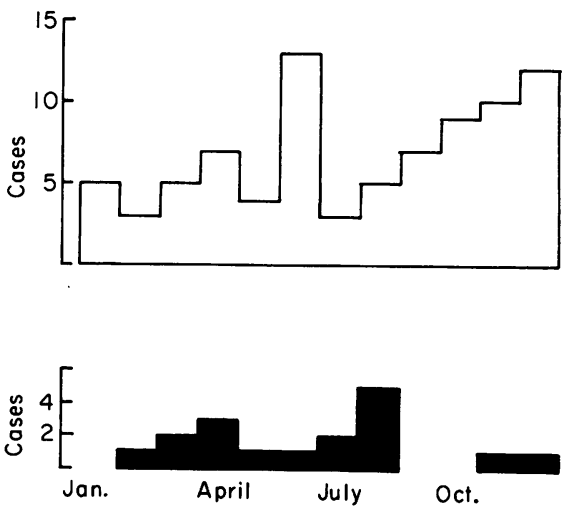

FIG. 2. Monthly incidence of wounding. Upper histogram: incidence of stab-wounds; lower histogram: incidence of other penetrating injuries. 
significant rise is observed in the winter months together with an unexplained peak in the month of June. Accidental penetrating injuries occurred most commonly in August, corresponding with school holidays, and was largely accounted for by a high incidence of child impalement injuries.

The time of arrival of stab-wounds in the Accident Room is shown in Fig. 3. It is seen that the majority occurred around midnight and in the early hours of the morning and only few during the daytime and evening. Other penetrating injuries took place sporadically throughout the day and night.

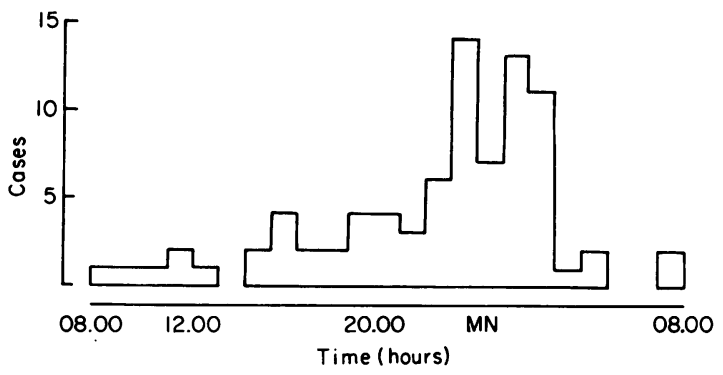

FIG. 3. Hourly incidence throughout the day of stabwounds.

The age distribution of the victims is given in Fig. 4. An obvious peak in stabbing injuries occurs in the 20-30-year-old age group. The age group with the greatest predisposition to accidental penetrating injury is the $0-10$-yearold group.
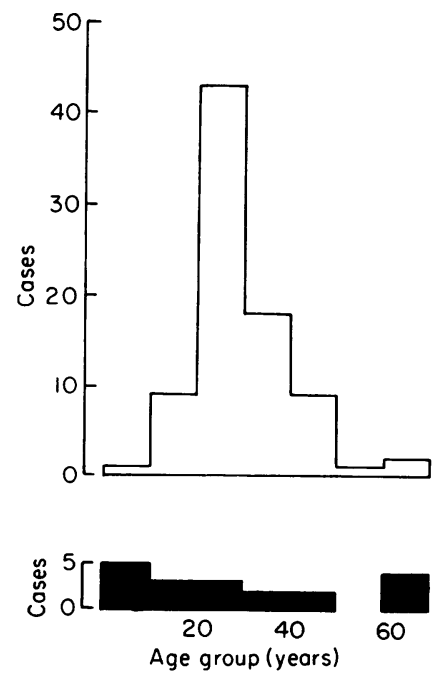

FIG. 4. Age group incidence of wounds. Upper histogram: incidence of stab-wounds; lower histogram: incidence of other penetrating injuries.

\section{Accidental injuries}

Of the 100 cases of penetrating injury reported, twenty-two were accidentally sustained. Of these three only were in females and were all caused by impalement. Males were injured in nineteen cases. Ten were due to boys falling on to a spike, fence or railing, and one adult impaled himself on railings in suspicious circumstances when he slipped whilst climbing in a built-up area in the dark at 11.30 p.m.

There were five accidental stab-wounds. Two butchers accidentally stabbed themselves whilst cutting meat and two further accidents occurred during horseplay with a knife. The fifth was suicidal.

There were four deaths, all occurring in males. Two of the deaths resulted from renal failure complicating peritonitis secondary to extensive penetrating injury of the abdomen sustained in road accidents. A third death resulted from haemorrhage when a motor cyclist, thrown from his vehicle, impaled himself on a fence, lacerating major vessels. The fourth was that of a suicide who stabbed himself in the heart.

\section{Assault injuries}

Seventy-eight per cent of all penetrating injuries were caused by criminal assault with aO $\overrightarrow{0}$ sharp instrument such as a knife. Over $60 \% \cong$. had evidence of alcohol excess which had obviously been a causal factor, and this applied equally to men and women involved in stabbing incidents. Three women claimed to have been accidentally stabbed at home, but circumstances were such that it was inferred that the woman's affection for the truth was less than that for her assailant.

There was an overall mortality in this group of patients of $6 \%$. This represented five deaths all of which were in men.

The majority of the victims were British, but over $30 \%$ occurred among the immigrant population. Amongst these the Irish and West Indian were most commonly involved, but Greek, Hungarian, Pakistani, Indian, Sikh and Poles were other nationalities represented. Two policemen were stabbed whilst on duty, and apart from a company director, a student and a B.B.C. employee, the others were of social class III and IV. Only one patient was unemployed.

Stab-wounds of the thorax and abdomen were single in $71 \%$ of cases and multiple in $29 \%$. They were caused by a variety of implement from scissors, carvers and a saw blade in domestic quarrels to penknives, sheath knives, screwdrivers and stillettos in other disputes. 
A high proportion of the stabbings occurred in the vicinity of public houses and clubs. They also occurred in or near the home: a man stabbed by his wife, son or cohabitant, or a woman by her husband or friend. Innocent bystanders were involved in four cases when trying to restrain or protect people.

In considering the clinical aspect of stabwounds, these injuries fall into three groups: abdominal wounds, thoracic wounds and a smaller thoraco-abdominal group. Results are reported under these headings.

\section{Abdominal wounds}

The numbers of abdominal and thoracoabdominal stab-wounds and their mortality are given in Table 1 .

TABLE 1

Numbers of abdominal/thoraco-abdominal/thoracic wounds and deaths

\begin{tabular}{lccr}
\hline $\begin{array}{c}\text { Position of } \\
\text { wound }\end{array}$ & $\begin{array}{c}\text { Stab- } \\
\text { wounds }\end{array}$ & $\begin{array}{c}\text { Other } \\
\text { penetrating } \\
\text { wounds }\end{array}$ & Total \\
\hline Abdominal & 34 & 7 & 41 \\
Deaths & 2 & 2 & 4 \\
Thoraco-abdominal & 9 & 0 & 9 \\
Deaths & 1 & 0 & 1 \\
Thoracic & 35 & 7 & 42 \\
Deaths & 2 & 1 & 3 \\
\hline
\end{tabular}

Of the forty-six abdominal wounds only one entered posteriorly and this pierced the abdominal aorta and was fatal. The remainder were distributed over the anterior abdominal wall as shown in Fig. 5.

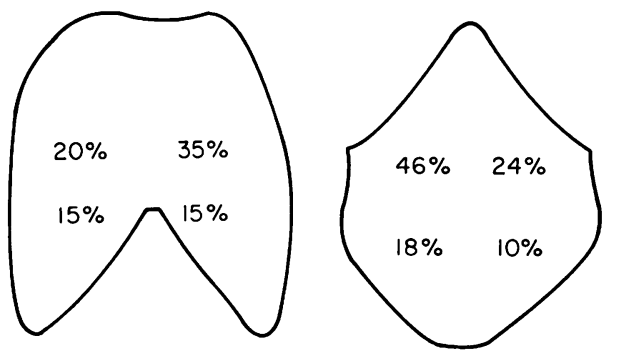

FIG. 5. Position of entry wounds in percentages in quadrants. Left: thoracic wounds; right: abdominal wounds.

\section{Clinical features}

The presenting symptoms and signs of intraabdominal injury are given in Table 2 .

Tenderness and guarding were consistently found to indicate intra-abdominal bleeding or organ injury, and when noted to be absent, in $20 \%$ of cases, laparotomy showed only peritoneal puncture.

TABLE 2

Incidence of abdominal signs on initial examination of cases where laparotomy was indicated and performed

\begin{tabular}{lc}
\hline \multicolumn{1}{c}{ Symptom or sign } & Incidence \\
\hline Tenderness and guarding present & $80 \%$ \\
Clinical shock absent & $63 \%$ \\
Bowel sounds present & $60 \%$ \\
Clinical shock present & $37 \%$ \\
Bowel sounds absent & $30 \%$ \\
Tenderness and guarding absent & $20 \%$ \\
\hline
\end{tabular}

Clinical shock was considered to be present when the pulse was more than $120 / \mathrm{min}$ and the blood pressure less than $100 \mathrm{mmHg}$, a transfusion of more than 1 pint of blood was required or when a patient was clinically noted to be pale, sweating and cold. This was always associated with injury to an abdominal viscus. Varying degrees of hypotension and tachycardia were encountered in inebriated patients even without organ injury. Even though vasodilation and a good peripheral circulation were present, alcohol was often a factor making clinical assessment difficult.

The presence of an upper abdominal wound with a shocked state was, on two occasions, due to intra-thoracic injury, the knife having pierced the peritoneum and diaphragm without injuring any abdominal organs.

Bowel sounds were present on initial examination in $60 \%$ and absent in $30 \%$ of cases of penetrating injury. It was not possible to correlate these figures with the absence or presence of any intra-abdominal injury as revealed by laparotomy. The sign was thus of very limited value.

Fifty per cent of abdominal wounds were in the right hypochondrium, presumably due to the termination of an upward stab to the abdomen by a right-handed assailant. This corresponded well with the high number of liver, diaphragm and stomach injuries (Table 3).

Wounds in the lower abdomen were much less frequent, and caused fewer visceral perforations, the small and large bowel being damaged infrequently and the bladder not at all.

Multiple stab-wounds were inevitably associated with organ injury.

\section{Investigations}

Patients who were clinically shocked had routine blood-grouping and cross-matching. 
Abdominal X-rays were found to be valueless in the assessment of abdominal injury. However, since two of the thoraco-abdominal injuries were originally thought to be solely abdominal and later on exploration found to be combined, it is suggested that an erect film of the chest is essential in upper abdominal stab-wounds to exclude haemothorax or pneumothorax.

TABLE 3

Incidence of organ injury in abdominal and thoracoabdominal stab-wounds

\begin{tabular}{lccc}
\hline \multicolumn{1}{c}{ Organ } & $\begin{array}{c}\text { Thoraco- } \\
\text { abdominal }\end{array}$ & Abdominal & Total \\
\hline Liver & 6 & 4 & 10 \\
Stomach & - & 6 & 6 \\
Diaphragm & 7 & - & 7 \\
Omentum & - & 3 & 3 \\
Ileum and mesentery & - & 2 & 2 \\
Colon and mesocolon & - & 4 & 4 \\
Pancreas & - & 2 & 2 \\
Duodenum & - & 1 & 1 \\
Spleen & - & - & - \\
Kidney & - & 3 & 3 \\
Inferior vena cava & 1 & - & 1 \\
Aorta & - & 1 & 1 \\
Retroperitoneum & - & 2 & 2 \\
Total & 14 & 28 & 42 \\
\hline
\end{tabular}

\section{Treatment and results}

Treatment of these forty-six abdominal stabwounds consisted of laparotomy in twenty-six $(56 \%)$. In twenty $(44 \%)$ definite organ injury was noted and required surgery but in the remaining six $(11 \%)$ no organ injury was noted, although the peritoneum was breached in four.

Fifteen patients $(33 \%)$ had a local exploration of the wound and this was considered to be adequate treatment at that time. Of the remaining five cases, four refused operative treatment and two discharged themselves. The remaining two and one other patient had no surgical procedure, were only observed, and were subsequently discharged without complication occurring.

The mortality in this series of abdominal stabwounds was $6.5 \%$.

The average length of stay of a patient with an abdominal wound was 13.4 days.

\section{Thoraco-abdominal stab-wounds}

No penetrating thoraco-abdominal wounds occurred other than those caused by assault with a knife. A notable feature of these stab-wounds was the association between uppper abdominal wounds and unsuspected thoracic injury. In three cases of thoraco-abdominal injury where there was a single wound presenting in the upper abdomen there was also thoracic injury and in one case the pericardium had been pierced. In two of the remaining seven cases in this group where the wounds were multiple, the main thoracic injury was caused by the extensive penetration of the abdominal wound.

No cases required thoracotomy for their chest injuries and only two had underwater-seal drainage tubes inserted. One patient died from a complication of his abdominal wound, namely a massive haemorrhage from a lacerated inferior vena cava.

\section{Thoracic stab-wounds}

There were forty-seven cases of stab-wounds of the thorax with two deaths (mortality $4 \%$ ). Eighty-five per cent of stab-wounds entered over the anterior chest wall and only $15 \%$ were situated on the back (Fig. 5).

\section{Clinical features}

Clinical signs and symptoms were assessed in cases of pneumothorax and haemothorax and are given in Table 4.

\section{TABLE 4}

Incidence of chest symptoms/signs on initial examination of cases where pneumothorax or haemothorax was present

\begin{tabular}{lc}
\hline \multicolumn{1}{c}{ Symptom or sign } & Incidence \\
\hline Painful respiration & $72 \%$ \\
Clinical shock present & $62 \%$ \\
Impaired respiration & $56 \%$ \\
Surgical emphysema & $48 \%$ \\
Clinical shock absent & $38 \%$ \\
Sucking wound & $28 \%$ \\
Cyanosis & $4 \%$ \\
Haemoptysis & $2 \%$ \\
Tension pneumothorax & 0 \\
\hline
\end{tabular}

Although these symptoms and signs when present give an indication of serious injury, their absence does not give corresponding reassurance. It was noted that cyanosis and haemoptysis were encountered rarely and no case of tension pneumothorax occurred.

Of the forty-five survivors, haemothorax was present in $21 \%$, surgical emphysema in $37 \%$ and radiological pneumothorax in $50 \%$

The position of the chest wounds (Fig. 5) shows a preponderance in the upper left chest (33\%) due to a downward stab by a righthanded assailant and illustrating the potential vulnerability of the heart to this type of injury. Of two surviving cases of cardiac injury included in this series, both presented with wounds over the left chest and evidence of a haemothorax. 


\section{Investigations}

These were confined to erect PA and lateral films of the chest and diagnostic needle aspiration in cases of suspected haemothorax or pneumothorax.

Electrocardiography was carried out in cases of suspected cardiac injury but was only of value in this series in following the recovery after cardiac injury.

\section{Treatment}

This was essentially conservative. Thirty-four patients had simple skin toilet and suture. They were observed and given antibiotics and either ATS or tetanus toxoid. In this group a small pneumothorax occurred in fifteen patients. One refused treatment. Apart from partial lobar collapse and some consolidation occurring in a few patients, there were no serious complications and none required further surgery.

One patient had a pneumothorax which was treated by aspiration.

Intercostal drainage tubes with underwater seal were inserted in nine patients. In six there was an ominously large or increasing pneumothorax and in three there was a haemopneumothorax. This group had a number of post-operative pulmonary complications such as partial collapse and consolidation. These resolved in all but one case which required aspiration of a loculated collection of fluid.

Thoracotomy was undertaken in three patients one for a suspected heart wound and two for deterioration while under observation. The latter two had persistant pulmonary bleeding which was controlled by simple suture. Decortication had to be performed later in one for a clotted haemothorax.

\section{Discussion}

\section{Abdominal stab-wounds}

The basis of treatment in this reported series of abdominal stab-wounds has been by immediate exploratory operation, either a full laparotomy, or, in certain circumstances, a local exploration. Initial local exploration of the wound track was undertaken in patients without signs, whose wound was sited over the costal margin, loin or perineum. Occasionally obviously superficial wounds on the anterior abdominal wall were explored, but this practice was potentially dangerous as a wound track is easily lost when it traverses rectus sheath and aponeurotic layers. Laparotomy was undertaken in all patients with abdominal signs and in all those without where there was a possibility of intraabdominal penetration, particularly where the wound was on the anterior abdominal wall. Of the few not treated in this way, three cases were merely kept under observation without mortality or morbidity. Two of these patients refused any operative treatment. The third had an apparently trivial wound. Two other patients with abdominal stab-wounds took their own discharge. As they were not re-admitted and do not appear in the City's Police records of deaths by stabbing it must be assumed that they recovered without ill-effect.

In assessing the value of clinical signs, the presence of clinical shock and significant tenderness and guarding were the only ones found to give a reliable indication of intra-abdominal injury. Of the investigations, abdominal $\mathrm{X}$-rays were found to be valueless, but $X$-rays of the thorax should be taken with every upper abdominal wound to exclude thoracic involvement. Further, in comatose or unrousable patients, or where a language difficulty exists, diagnostic paracentesis may be of limited use (Morris, 1966). Local injection of radio-opaque material into the wound-track has also been used to define whether the peritoneum has been breached and reports have been satisfactory (Rodkey, 1966). Neither of these techniques has been used in this series.

Treatment of individual organ injury was straightforward and agrees with that described by other authors (Roberts \& Lavelle, 1966 ; Goldman, de Laurentis \& Rosemond, 1962). Stomach and small bowel perforations were sutured; large bowel perforations were also sutured without colostomy, but colostomies were made for rectal wounds. Liver injuries were sutured or had stopped bleeding at laparotomy. They were all drained. Pancreatic injuries were sutured and drained. Injuries to the extra-hepatic biliary system and spleen were not seen.

A mortality of $6.5 \%$ is comparable with that of American authors (Rodkey, 1966: 6.2\% ; Goldman et al., 1962: 3.6\%). Two cases died early with asystolic cardiac arrest following haemorrhage from the aorta and inferior vena cava, and the other died later from renal failure complicating peritonitis. The mortality from large vessel haemorrhage is invariably high; a figure of $70 \%$ is given by Beall (1960), and of $75 \%$ by Parmley, Mattingley \& Mannion (1958). Prompt resuscitation and early surgery offer the only hope, for these patients' deaths occur quickly following the injury. A high degree of suspicion from the nature and position of the injury in a relatively alert patient is required.

The results of treatment in this series would support an operative approach to the manage- 
ment of the condition. No deaths resulted directly from operative measures or from failure to operate when indicated. Again no significant morbidity occurred with this method of treatment.

Recently Shaftan (1960) and Rothschild \& Treiman (1966) have recommended a conservative policy of selective management in an effort to reduce the numbers of abdominal stabwounds requiring operative treatment and also to reduce their length of hospital stay. Cases with clinical evidence of intra-abdominal injury had a full surgical exploration and those without were observed. They point out that several of their cases of stab-wounds were first seen several days after injury without any evidence of peritonitis. These cases were observed and were discharged earlier than would have been possible if laparotomy had been performed. The results of selective management are claimed to show a reduced morbidity with no increase in mortality and that average length of hospital stay was reduced. A conservative policy of selective management is based on the reliability of clinical signs as an indication of intra-abdominal injury. Only tenderness and guarding were noted to be reliable signs in our series and clinical assessment of the degree of intra-abdominal or thoraco-abdominal injury was in error on some occasions. A conservative approach to treatment in some of these cases would have been hazardous and would not have provided any distinct advantages over the above operative approach. Any patient who has a stab-wound and has exploration deferred is a source of continual worry. Certainly any reduction in mortality must be by earlier assessment, resuscitation and operation.

\section{Thoracic stab-wounds}

The treatment of stab-wounds of the thorax in this series, in contrast to that of abdominal wounds, was essentially conservative. The fact that few complications occurred either during or after treatment supports such a policy which is based on clinical and radiological assessment and regular observation. The presence of fluid or a significant degree of air (greater than 20\%) in the pleural cavity was treated with intercostaltube drainage with immediate satisfactory results and no long-term complications. This method is to be preferred to thoracocentesis in that the evacuation of blood and air is more complete and any persistent bleeding is more quickly diagnosed when an intercostal drainage tube is present. Two cases in this series treated in this way gave an early indication of massive bleeding and subsequently underwent thoracotomy. Again there was a significant number of minor pulmonary complications. Residual radiological $\stackrel{\mathbb{Q}}{\varrho}$ pleural thickening was not considered as a com- $c$ plication. There were no empyemata and no significant incidence of pleural infection with this $\stackrel{5}{+}$ form of chest drainage, contrary to the experience in the Korean war (Valle, 1952).

Thoracotomy was undertaken for a stabwound of the heart and in the above cases of intra-pleural bleeding. One of the latter required later decortication for a clotted haemothorax (Nicholson \& Scadding, 1944), the only case where later elective surgery was required.

TABLE 5

Incidence of organ injury in thoracic and thoraco-abdominal stab-wounds

\begin{tabular}{lccc}
\hline Organ & $\begin{array}{c}\text { Thoraco- } \\
\text { abdominal }\end{array}$ & Thoracic & Total \\
\hline Lungs & 6 & 22 & 28 \\
Diaphragm & 7 & 0 & 7 \\
Pericardium & 1 & 3 & 4 \\
Heart & 0 & 3 & 3 \\
Great vessels & 0 & 0 & 0 \\
\hline
\end{tabular}

The mortality of two $(4.4 \%)$ in this series was caused by cardiac injury or injury to the $\vec{\varphi}$ major thoracic vessels. Both patients died in the Accident Room, minutes after admission, fron $\bar{Q}$ asystolic cardiac arrest associated with tampon $F$ ade. Three other patients, not included in this series, were found to be dead on arrival as a result of a cardiac stab-wound, and at necropsy were found to have a large amount of blood in the pericardium. While death is probably inevitable in a large number of cases of cardiac injury by stabbing (only twelve surviving cases are recorded in the British literature) the two surviving cases here and those of others (Dickenson, 1965 ; Crymble, 1961 ; Lawrie, 1955 ; Pryer, 1953) encourage attempts at vigorous resuscitation with pericardiocentesis and immediate thoracotomy.

\section{Acknowledgments}

I would like to thank H.M. Coroner, Mr D. Summerfield, the Chief Constable for Manchester and the Medical Board of the United Manchester Hospitals for permission to review cases. Acknowledgment is gratefully made to $\mathrm{Mr} \mathrm{N}$. C. Keddie and Mr W. F. Nicholson for their help in the preparation of this paper, and to the Medical Illustration Department of the Manchester Royal Infirmary for the histograms.

\section{References}

Beall, A.C. (1960) Penetrating wounds of the aorta. Amer. J. Surg. 99, 770.

CRYmble, B. (1961) Stab-wounds of the heart. Brit. J. Surg. 48, 651 . 
Dickenson, K.M. (1965) Multiple stab-wounds including injury of the heart. Proc. roy. Soc. Med. 58, 1081.

Editorial (1966) Stab-wounds of the trunk. Brit. med. J. i, 1437.

Goldman, L.I., DE LAURenTIS, D.A. \& Rosemond, G.P. (1962) Penetrating abdominal wounds in a civilian population. Amer. J. Surg. 104, 46.

LAWRIE, R.W. (1955) Recovery after penetrating wound of the heart; case report. Brit. med. J. i, 952.

MORRIS, P.J. (1966) Diagnostic paracentesis of the acute abdomen. Brit. J. Surg. 53, 707.

Nicholson, W.F. \& Scadding, J.G. (1944) Penetrating wounds of the chest; a review of 291 cases in the Middle East. Lancet, i, 299.
Parmley, L.F., Mattingley, T.W. \& Mannion, W.C. (1958) Penetrating wounds of the heart and aorta. Circulation, $17,953$.

PRYER, R.R.L. (1953) Penetrating heart wound; value of intra arterial transfusion. Lancet, ii, 327.

Roberts, G. \& LAVELle, E. (1966) The management of stabwounds of the abdomen and thorax. Brit. J. Surg. 53, 88.

RODKEY, G.V. (1966) The management of abdominal injuries. Surg. Clin. N. Amer. 46, 627.

Rothschild, P.D. \& Treiman, R.L. (1966) Selective management of abdominal stab-wounds. Amer. J. Surg. 111, 382.

ShafTAN, G.W. (1960) Indications for operation in abdominal trauma. Amer. J. Surg. 99, 657.

VAlle, A.R. (1952) Management of war wounds of the chest. J. thorac. Surg. 24, 457. 